\title{
Blur and interoceptive vision
}

\author{
Błażej Skrzypulec ${ }^{1}$ (1)
}

\begin{abstract}
The paper presents a new philosophical theory of blurred vision according to which visual experiences have two types of content: exteroceptive content, characterizing external entities, and interoceptive content, characterizing the state of the visual system. In particular, it is claimed that blurriness-related phenomenology interoceptively presents acuity of vision in relation to eye focus. The proposed theory is consistent with the representationalist thesis that phenomenal character supervenes on representational content and with the strong transparency thesis formulated in terms of mind-independentness. Furthermore, the interoceptive approach is free from controversial assumptions adopted by other philosophical theories of blurred experiences and is able to account for the epistemic and motivational role of visual blur, i.e. that blurred experiences provide a prima facie justification for beliefs regarding our vision and motivate actions directed toward our eyes.
\end{abstract}

Keywords Blur - Vision - Interoception - Exteroception - Representationalism · Transparency

The phenomenon of blurred vision has been interesting for philosophers of perception for two major reasons. First, blurred visual experiences are problematic for the representationalist thesis that perceptual phenomenal character supervenes on representational content (see Nanay 2018; Schroer 2002; Tye 2003). For instance, when one takes off glasses, vision may become blurred. This is clearly a change in visual phenomenal character, but it is far less obvious whether this change

Błażej Skrzypulec

blazej.skrzypulec@uj.edu.pl

1 Institute of Philosophy, Jagiellonian University, Kraków, Poland 
is associated with any modification regarding which objects and properties are visually represented. It may seem that after taking off glasses the same entities are represented, but are experienced in a phenomenally distinct, blurry way. Second, blurred vision is invoked as a counter-example against the transparency thesis, i.e. a position that in perceptual experiences we are aware only of mind-independent objects and properties (see Boghossian and Velleman 1989; Pace 2007; Smith 2008). It is so because in a blurred experience, blurriness does not seem to be a property attributed to perceived, external objects. In fact, many philosophical theories of blurred vision weaken or abandon the transparency thesis by postulating that blurriness is a property of some mental entities (e.g., Crane 2006; Pace 2007) or discard the intuition that in blurred experiences blurriness is not attributed to external objects (e.g., Allen 2013; Gow 2019).

This paper presents a novel theory of blurred vision which is consistent with both representationalism and transparency. To account for blurred vision, I propose, relying on important similarities between blurred experiences and interoceptive states such as bodily sensations, that visual experiences have two types of content: (a) exteroceptive content, which characterizes external entities; and (b) interoceptive content, which characterizes a state of the visual system. When vision becomes blurred, as in case of taking off one's glasses, some exteroceptive content is lost and visual system is interoceptively presented as having lower acuity. More specifically, I argue that there is a variety of blurred experiences which present different distributions of acuity in relation to eye focus.

Such a theory is consistent with the representationalist thesis, as when vision becomes blurry, there is associated modification in interoceptive visual content. It also preserves the transparency thesis, as while blurriness is not attributed to external objects, it is also not attributed to mental entities, but to the visual system, which is a material being, no less mind-independent than dogs, chairs, and tables. Furthermore, the proposed theory accommodates the fact, often neglected in philosophical conceptions of blurred vision, that blurred experiences provide a prima facie justification for beliefs regarding our vision and motivates actions concerning our eyes (like rubbing, blinking, or squinting).

In Sect. 1, I explicate the key notions used in the paper such as 'blurriness' and 'fuzziness,' the distinction between 'global blur' and 'depth blur,' and alternative formulations of the transparency thesis. Subsequently, in Sect. 2, I review the contemporary philosophical theories of blurred vision in order to point out their controversial assumptions. Finally, Sect. 3 is devoted to presenting the interoceptive theory of blurred vision.

\section{Blurriness, transparency, and representationalism}

In philosophical works, the phenomenology of blurred experiences is usually distinguished both from that of clear experiences and from phenomenology associated with perceiving entities as being fuzzy (see Crane 2006; Smith 2008; Tye 2003). For instance, the example of taking off glasses demonstrates the distinction between clear and blurred experiences. Without glasses, the phenomenal character 
of an experience seems to be different, but it is less obvious whether there is also any change in representational content (and so problem for the representationalist thesis arises) and blurriness does not seem to be a property of perceived entities (which generates a problem for the transparency thesis). However, even a clear experience can present some entities as being fuzzy, i.e. as having indistinct edges. For example, this frequently happens when perceiving clouds, shadows, or watercolor paintings. The phenomenology associated with clearly perceiving fuzzy objects seems to be distinct from that associated with perceiving sharp edges blurrily and, as in the eyeglass example, it is not obvious whether this phenomenal distinction corresponds with a difference in representational content. Furthermore, when a fuzzy edge is perceived clearly, fuzziness seems to be a property which is visually attributed to an external entity. In contrast, when a sharp edge is perceived blurrily, it is less plausible to postulate that blurriness is externally attributed as a property of the edge. Experiencing objects in a blurry way is also distinguished from experiencing objects by using peripheral vision. In both cases, the surroundings are presented in a less detailed manner than is the case for more usual clear experiences, but philosophers generally agree that the phenomenology of peripheral vision is distinct from that of blurred experiences (see Allen 2013; Nanay 2018). In consequence, visual blur is considered as a phenomenon concerning central vision.

A further distinction can be made within the category of blurred experiences itself. First, a blurred experience may result from a general drop in acuity such that all things within visual field become blurry no matter the eyes' current focus. This is usually a sign of disorder caused by, inter alia, fatigue, dry eyes, myopia, intoxication, or physiological disturbances within the visual cortex. Because this form of blurriness encompasses whole visual field, I will refer to such blurred experiences as involving 'global blur.' Second, blurred experiences commonly occur due to variations in how eyes are focused. When one focuses on a proximal object, then distant objects are experienced blurrily; conversely, focusing sight on distant objects makes nearby items appear blurry. Different than in case of global blur, this type of visual blur does not encompass the whole visual field, as while some objects look blurry, others are perceived clearly. Blurred experiences of this type are not associated with any disorder and blurriness resulting from variations in eye focus serve as a cue for mechanisms responsible for depth perception, leading me to name this form of blur 'depth blur.' In fact, the majority of visual experiences which we intuitively consider 'clear' involve some phenomenal combination of clearness and depth blur, as not all objects are perceived with the same acuity due to way in which eyes are focused.

As I have already stated, the phenomenon of blurred vision is usually discussed in the context of representationalism and transparency. There seems to be widespread agreement that the relevant representationalist thesis is that phenomenal character supervenes on representational content, or, in other words, that every change in phenomenal character is associated with a change in content (see Bourget 2015; Nanay 2018; Schroer 2002; Tye 2003). However, such general agreement is not present in case of the transparency thesis. The basic intuition concerning transparency is that we are aware only of mind-independent objects and their properties in our visual experiences. In other words, according to the transparency 
thesis, we do not need to postulate the awareness of any mental elements such as qualia or properties of the experience itself to account for the phenomenology of experiences. Transparency is often accepted together with representationalism because a thesis that we are aware only of mind-independent objects and their properties may seem to be in favor of a thesis that phenomenal character supervenes on content. However, the connection between transparency and representationalism is not necessary, as it is possible to postulate that phenomenal character supervenes on content while specifying content in such a way that it characterizes also some nonstandard, mental entities (see Pace 2007 for such a theory).

The transparency thesis is sometimes given a weaker, phenomenal reading according to which it seems to us that we are only aware of presented objects and properties. However, a stronger, metaphysical understating is also adopted, which states that we are in fact only visually aware of presented objects and properties (see Gow 2019 for this distinction). Furthermore, in order to distinguish them from entities such as qualia, presented objects and properties which transparency holds we are aware of are sometimes characterized as 'mind-independent entities' (e.g., Allen 2013; Crane and French 2015, 2015; Martin 2002) or alternatively as 'external entities' (e.g., Tye 2002).

Mind-independentness is postulated to exclude mental entities and their properties. However, externality may be understood in a stronger way as excluding not only mental entities but also our body and its parts. My interoceptive theory of blurred experiences is consistent with both weaker phenomenal and stronger metaphysical readings of the transparency thesis. Nevertheless, because I interpret visual blur as presenting state of visual system, my account does not satisfy these formulations of the transparency thesis which characterize transparency in terms of externality, but only those which use the notion of mind-independence. While the visual system is a non-mental, physical entity which possesses physical properties, it is obviously an internal fragment of the perceiver's body. ${ }^{1}$ However, even if one treats the formulation of the transparency thesis made in terms of externality as the correct one, the fact that may account explains the phenomenon of blur without reference to mind-dependent entities may still be considers as its advantage.

\section{Theories of blurred vision}

In this section, I discuss the contemporary philosophical theories of blurred vision. I do not aim to provide arguments which would force anybody to abandon these theories, but I show that all available accounts of blurred experiences have some important drawbacks. This serves as a basis for showing that the described problems do not threaten my interoceptive theory. First, I present accounts which preserve both the representationalist thesis and the transparency thesis at the cost of

\footnotetext{
1 One may claim that visual system is also a mental entity, as it is one of the constituents of the mind. However, it is not the sense of 'mental' that is relevant here. The visual system is non-mental and mindindependent because, unlike qualia, is a physical, spatiotemporal entity that is not produced by mental activities, but itself plays a role in generating mental states.
} 
sacrificing the intuition that blur is not visually attributed to external objects. Second, accounts which accommodate this intuition but reject or weaken the transparency thesis are discussed. Finally, I describe negative theories of blurred vision that analyze blurred experiences in terms of loss of content. I believe that negative theories present correct but incomplete account of visual blur which should be supplemented by introducing interoceptive visual content.

\subsection{Blur as a property of external objects}

A common theoretical move is to explain blurred vision in a way that is consistent both with the representationalist thesis and the transparency thesis at the cost of rejecting intuition that visual blur is not a property attributed to external objects. For instance, Nanay (2018) provides such an account of blurred experiences by referring to the distinction between determined properties, like a particular shade of red with specified saturation and brightness, and determinable properties like the color red in general. According to his proposition, in blurred experiences objects are perceived as not having fully determined properties concerning the location of their edges. ${ }^{2}$ This differentiates blurred vision from clear vision, including clear vision of fuzzy items, in which determined properties are attributed to objects. In consequence, transparency is saved, as in blurred experiences we are aware of determinable properties of mind-independent objects, and representationalism is preserved, as phenomenal differences between blurred and clear vision correspond to the distinction between presenting determinable and determined properties.

However, it less obvious whether Nanay's proposal is able to distinguish blurred vision from peripheral vision. Nanay is aware of this problem and claims that in blurred vision only properties concerning the localization of edges are presented as determinable but that in peripheral vision all properties, like colors or shapes, are not fully determined. However, such a criterion does not seem to work properly in case of heavily blurred experiences, where a variety of properties, like those concerning shape or size, are experienced in indeterminate ways. Even if this problem can be amended, I believe that abandoning the intuition that visual blur is not attributed to external objects constitutes a significant cost. In particular, visual blur does not seem to play the same epistemic role as usual visual properties. Typically, visually presenting an object as being $F$ serves as a prima facie justification for a belief that the considered object is $F$. This means that having an experience as of an object as being $F$ justifies that the object has the considered property, but this justification can be defeated by some additional evidence-for instance, suggesting that our visual state is illusory (see Siegel and Silins 2015 for a review). On the contrary, it is less plausible to accept that perceiving something blurrily provides a justification that this thing in fact has blurry edges. Instead, it leads to beliefs concerning the state of our vision. In particular, when vision

\footnotetext{
${ }^{2}$ It should be noted that according to Nanay blurred vision involves an attribution of determinable properties to external objects and not just a loss of some determinate content (see Nanay 2018, p. 4). This differentiates Nanay's approach from negative theories of visual blur (see Sect. 2.3).
} 
suddenly becomes globally blurred, a person is likely to believe that her vision is not working properly. ${ }^{3}$

A proponent of a thesis that visual blur is attributed to external objects may try to explain why there is a strong intuition that such external attribution does not occur. One idea is to argue that blur is visually attributed to external objects, but the contrary intuition arises due to peculiarities of the content of blurred vision. For instance, Allen (2013) formulates the 'over-determination' theory, according to which blurred experiences have internally inconsistent content as they present edges of objects as being simultaneously located at many distinct places. ${ }^{4}$ Such an inconsistent nature of visual blur may be a source of intuition that blur is not visually attributed to external objects. However, this proposal is not without serious controversies. From the phenomenal perspective, it is doubtful whether blurred experiences present the environment in an internally inconsistent fashion. In particular, blurred experiences are introspectively quite different from the experiences usually invoked to demonstrate cases of self-contradictory visual content. For instance, in the waterfall illusion it seems that something is both moving and not moving, and in Escher's drawings, an edge seems to be both in front and behind some other edge. However, blurred vision intuitively seems to present entities not as having incompatible properties but rather as being presented in a low-detail way. Furthermore, even if blurred vision presents edges as simultaneously multiply located, there is not necessarily anything internally inconsistent in such multiple localization. In fact, the ability to be simultaneously multiply located is often invoked in metaphysical works in order to distinguish universals, which can be multiply located, from particulars, which cannot, and is treated as an internally consistent property (e.g., Daly 1994; Heil 2003, pp. 132-136).

Another way of 'explaining away' the intuition that blur is not visually attributed to external objects is through denying the claim that the perceptual phenomenology of blurred experiences is distinct from the phenomenology of clear experiences presenting fuzzy items. In particular, Gow (2019) has proposed that blurred experiences, but not experiences of fuzzy things, are accompanied by nonperceptual 'intellectual seemings' due to which it seems to a subject that blur is not a property of external entities. The occurrence of such seemings may be a source of intuition that blur is not visually attributed to items in the environment. However, according to Gow, the strictly perceptual phenomenology of blurred experiences is

\footnotetext{
3 It should be noted that the thesis that visual blur justifies beliefs about one's vision is consistent with a claim that such justification is primarily provided by experiencing objects in a blurry way (mainly because visual blur is less apparent in case of empty visual space). For instance, it may be the case that I have a hallucinatory experience as of a blurry object. In such a case, learning that I do not experience an object also means learning that I do not experience an object in a blurry way (as there is simply no object to be experienced in any way). In consequence, realizing that an experience is a hallucination may counter a prima facie justification regarding the state of one's visual system provided by visual blur.

4 Another idea regarding the peculiarity of blurred experiences' content has been proposed by Pautz (2010), who maintains a non-predicational account of visual blur. According to this approach, blurred experiences present that there is blur everywhere without attributing blurriness to any particular object or place. However, such a position is still problematic as, contrary to the considered intuition, it assumes that visual blur is experienced as a feature present in the external environment.
} 
the same as perceptual phenomenology of clear experiences of fuzzy items, and so both of these experiences perceptually present external entities as having the same properties.

To justify that perceptual 'blurry phenomenology' is the same as perceptual 'fuzzy phenomenology,' Gow presents a thought experiment in which some participants look at a sharp picture through glasses which makes vision blurry while others look at a fuzzy picture through clear glasses. She claims that by relying solely on the phenomenology of perceptual experiences, participants would not be able to determine whether they are looking at a sharp or a fuzzy picture. Nevertheless, such an argument is inconclusive, as it does not follow from the fact that under certain circumstances vision does not phenomenally distinguish between some properties $F$ and $G$ that it generally cannot phenomenally distinguish $F$ from $G$. For instance, circular shapes and elliptical shapes are phenomenally distinct visual properties despite the fact that when objects are perceived from a certain perspective an experience of a circle can be phenomenally the same as an experience of an ellipse. Furthermore, it is not obvious whether in the example above one cannot introspectively distinguish seeing blurrily from seeing a fuzzy image by relying on perceptual phenomenology. For instance, changing eye focus is likely to introduce larger phenomenal changes in a case of seeing a fuzzy image than in a case of wearing blur-inducing glasses, as such glasses introduce a global blur that is independent from variations in eye focus. In consequence, it is likely that when perceiving a fuzzy image one can manipulate the indistinctness of its edges by eye movements to a greater degree than when vision is globally blurred.

A more general argument has been formulated by Schroer (2002), who claims that we distinguish between blurrily presented edges and clearly perceived fuzzy edges not because they phenomenally look differently but by comparing them with the rest of the visual field. For example, if edges look blurry in the whole visual field, it is a cue that one is in fact having a blurred experience. On the other hand, if a blurry edge is surrounded by clear edges positioned at the same distance, it is more likely that it is a clear experience of a fuzzy item. However, the fact that vision engages in such comparative heuristics does not constitute a sufficient justification for a thesis that 'blurry phenomenology' is the same as 'fuzzy phenomenology.' In fact, it may be that the phenomenology of blurred vision is distinct from phenomenology of vision presenting fuzzy objects exactly because the phenomenal differences are partially determined by the results of comparative procedures conducted by visual system. Of course, debates concerning phenomenal sameness and difference are difficult to settle, and I believe that one may coherently continue to maintain that visual blurriness is phenomenally the same as visual fuzziness. My point is that it is a controversial assumption; in Sect. 3, I show that one can explain the phenomenon of blurred vision in a way that is consistent both with the representationalist and transparency theses without identifying 'phenomenal blurriness' with 'phenomenal fuzziness.' 


\subsection{Abandoning transparency}

The theories of blurred vision discussed above maintain representationalism and transparency by rejecting the intuition that blur is not visually ascribed to external objects or by making some controversial theoretical moves to 'explain away' this intuition. There is also another class of theories which preserve intuition about the lack of external attribution of blur but postulate that to properly account for blurred vision we should abandon or severely weaken the transparency thesis. According to such theories, visual blur is a property of a mental item such as a subjective visual field or a visual experience itself (Bach 1997; Boghossian and Velleman 1989; Crane 2006). Often, the rejection of the transparency thesis is accompanied by rejection of the representationalist thesis, and visual blur is interpreted as a nonrepresentational phenomenal property (see Smith 2008). However, it is also possible to explain blurred vision in a way that weakens transparency but accepts the representationalist thesis. The most developed variant of this approach has been proposed by Pace (2007), who adopts a 'layered' notion of visual content. According to this position, visual experiences have two types of content: primary content, which characterizes external entities, and secondary content, which characterizes visual field. Thus, the representationalist thesis is saved, as the phenomenal transition from clear vision to blurred vision is associated with a modification of secondary content. Furthermore, this result is achieved without rejecting the intuition that blur is not visually attributed to external objects, because visual blur is treated as a property of a special, mental object: the visual field. However, by Pace's own admission (Pace 2007, pp. 331-332), his solution only allows maintenance of a very weak version of the transparency thesis, stating that it sometimes seems to us that we are aware of properties of mental entities but in usual situations the properties of mental entities are difficult to notice. According to usual phenomenal formulation of the transparency thesis it is not only difficult, but it never seems to us that we are aware of mental entities and their properties. Similarly, according to the usual metaphysical formulation of the transparency thesis, we are in fact never aware of mental entities and their properties, but in Pace's solution, we are always visually aware of some properties of mental visual field, even if it is usually difficult to notice this fact.

Alternatively, blurred vision is characterized not in terms of properties of mental entities but by referring to the notion of modes or ways of presentation (see Dretske 2003). This idea is often proposed in the context of considerations regarding differences between perceptual modalities. For instance, while both visual and tactile modality may present the same property, like roundness, and so share content, each modality presents content in a phenomenally distinct way (see Martin 1993; Richardson 2010). Applying the same idea to the problem of blurred vision allows a statement that transition between clear and blurred vision consists of representing the same properties under a different mode of presentation. Such a solution preserves the intuition that blur is not visually attributed to external objects, but is costly, as it entails some modifications regarding both representationalism and transparency. It is so because (a) there are some phenomenal changes, regarding modes of presentation, which may happen without changes in content, and (b) we 
are visually aware not only of mind-independent objects and properties but also of ways in which these entities are presented. Furthermore, explaining blurred vision in terms of modes of presentation is controversial, as according to usual understating, a mode of presentation constitutes a way in which the perceptual system gains access to some aspects of the environment. For instance, both vision and touch have some specific ways of accessing the property of roundness. However, blurred vision seems to be a phenomenon which does not reflect a perceptual ability to obtain some information, but also indicates an inability to access some data due to lowered acuity. Later, I argue that my interoceptive account of blur is free from such theoretical costs. In particular, is consistent both with the usual metaphysical and phenomenal reading of transparency, according to which we are in fact only visually aware of mind-independent objects and properties and it seems to us that we are only aware of such objects and properties.

\subsection{Blur as a loss of content}

Finally, there are theories which characterize blurred vision in purely negative terms, as a loss of visual content. This idea has been considered by Tye (2003) and further developed by Bourget (2015), who proposed an 'amplification argument.' This argument relies on a plausible assumption that blurred vision is a gradable phenomenon: one experience may be blurrier than another. Given that, we may consider a series of experiences such that first is clear and subsequent experiences are more and more blurred. It seems obvious that in the case of the last elements of such a series the majority of visual content is lost in comparison to the first, clear experience, as when blur is severe one is only aware of colorful, indistinct blobs. In consequence, a loss of representational content starts to occur somewhere between clear experience and severely blurred experience. However, it seems arbitrary to judge that up to some point in the middle of the series, perhaps the fifth or tenth element, no content is lost, but after that content starts to disappear. Instead, all occurrences of visual blur are associated with some loss of content which becomes more and more severe as the vision is more blurred.

I believe that Bourget's argument is convincing, and that blurred vision indeed involves loss of visual content. However, I do not think that a negative theory provides a full account of blurred vision. First, the negative theory does not distinguish between blurred vision and other phenomena which also plausibly involve loss of visual content. For instance, when an object is at first perceived by central vision and then by peripheral vision some content seems to be lost. However, blurred vision and peripheral vision are usually treated as distinct phenomena. Similarly, visual content may be gradually lost with changing environmental conditions, in particular when the amount of light in the surrounding is gradually falling. Second, the phenomenon of blurred vision has also certain positive aspects that are not accounted for by the negative theory. For instance, an occurrence of visual global blur seems to provide justification for a belief such as "something is wrong with my vision" and motivates actions such as blinking or rubbing eyes. In the next section, I rely on these positive aspects in developing an interoceptive account of blurred vision. 


\section{Interoceptive blur}

The theories described in the previous section characterize blurred vision by adapting some controversial assumptions. In particular, they severely weaken the transparency thesis, reject the intuition that blur is not attributed to external objects, characterize the content of blurred experiences as inconsistent, or claim that there is no phenomenal difference between blurred experiences and experiences presenting fuzzy items. Furthermore, virtually all of those theories do not recognize the positive aspects of blurred vision, i.e. that visual blur provides justification for beliefs about our visual system and motivates actions directed toward the eyes. In particular, when vision becomes globally blurred, it inclines us to believe that our vision is not working properly and we are likely to engage in activities, like blinking, intended to restore visual acuity. I believe that no philosophical theory of blurred vision can be complete without accounting for these peculiar features of blurred experiences. Of course, it should be noted that reflection concerning the positive aspects of visual blur is not completely absent in philosophical theories of blurred vision. For instance, Schroer (2002) recognizes that perception of sharpness of edges is importantly determined by the "resolving power of the visual system" and Smith (2008) claims that the source of intuition that blur is not attributed to external objects is that we know that blur results from a malfunction of the visual system. Furthermore, French (2015) observes, from the perspective of relational theories of perception, that a difference between clear and blurred vision consist in the fact that in case of blurred vision, a subject does not have access to some environmental properties due to her internal state. Nevertheless, these observations play only a peripheral role in major theories of blurred vision and do not lead authors to recognize the interoceptive dimension of visual blur.

\subsection{Blur and interoceptive experiences}

In contrast, I propose that the positive aspects of visual blur can be accommodated within a theory of blurred vision by utilizing the notion of 'interoceptive experiences,' i.e. experiences that present states of our own bodies. ${ }^{5}$ This category includes bodily sensations, like pain or tactile experiences, which are experienced as localized within bodily boundaries (e.g., Smith 2011; de Vignemont and Massin 2013); proprioceptive sensations, which concern the position of bodily parts (e.g., Elder 2013; Hochstetter 2016); and bodily feelings, like fever or tiredness (e.g., Slaby 2008), which are not experienced as localized within bodily parts but rather seem to concern the body as a whole.

While the category of interoceptive experiences is significantly diverse, such experiences have some characteristic features which differentiate them from usual exteroceptive experiences presenting external objects. First, their phenomenal character is such that it provides a prima facie justification for beliefs concerning

\footnotetext{
${ }^{5}$ For the purpose of this paper, I assume that interoceptive experiences can be understand as a type of perceptual experience. See Avila 2016; Aydede 2017; Fridland 2011; Schwenkler 2013 for philosophical discussions concerning this issue.
} 
our own body (see Bain 2003; Mattens 2016; O'Sullivan and Schroer 2012; de Vignemont 2017 for different expressions of this intuition). Even in cases of some severe interoceptive illusions or hallucinations, like when one feels pain in a phantom limb or experiences a tactile sensation during the rubber hand illusion, the sensation is experienced as concerning one's body and provides initial justification for a false belief concerning bodily states. However, the phenomenology of exteroceptive experiences is silent regarding whether or not a presented object is part of one's body. For instance, a purely visual phenomenology of seeing one's own hand may be exactly the same as the phenomenology of seeing, appropriately positioned, someone else's hand. In consequence, while exteroceptive experiences may sometimes provide justification for beliefs concerning our body, such justification is not provided simply in virtue of the phenomenal character of exteroceptive experiences.

Second, compared to exteroceptive experience, interoceptive experiences usually have a stronger motivational force, i.e. they often incline one to engage in certain actions. In particular, pain and thermal experiences often evoke some avoidance behaviors (see Klein 2012; O'Sullivan and Schroer 2012). This point can also be demonstrated for tactile experiences, which are commonly treated as having both exteroceptive and interoceptive character (e.g., Mattens 2013; Ratcliffe 2008; Richardson 2011). It is so because tactile experiences present some properties of external entities, for instance, that something is round, and also inform one about the state of the body, for example that there is pressure exerted on a fragment of the skin. The exteroceptive aspects of tactile experiences are not particularly associated with motivational force. For instance, tacitly experiencing something as round or heavy does not strongly incline one to conduct any actions. However, tactile experiences with salient interoceptive component-for instance, when something is crawling on the skin's surface-often motivate avoidance behaviors (see Mattens 2016). Similarly, visceral states such as hunger typically motivate actions directed toward satisfying certain desires. In contrast, though, while exteroceptive experiences provide information about actions that can be undertaken, for instance by presenting shape of an object, they usually lack salient motivational force.

I believe that the features outlined above are also possessed by many blurred visual experiences. First, as already observed, there is a strong intuition that the phenomenology of visual blur does not suggest that blurriness is visually attributed to external objects. In other words, blurred experiences do not provide justification for a belief that the world itself is blurred. Instead, the phenomenology of blur is such that it intuitively provides a prima facie justification for beliefs concerning the state of our visual system. In particular, having globally blurred vision justifies a belief that something is wrong with our vision and a presence of depth blur may justify that our eyes are focused on a nearby, or a distant, object. Of course, the specific content of a belief justified by visual blur depends in an important way on context and background knowledge. For instance, a person with scientific knowledge concerning perception may literally form a belief referring to her visual system and to a specific disorder. However, in different circumstances, the justified belief may refer to eyes, which are commonsensically the parts of our body responsible for seeing, and to some unspecified disorder (e.g., "Something is wrong 
with my eyes!") or simply to the ability to see without characterizing in virtue of what this ability is realized (e.g., "I cannot see properly!"). Nevertheless, despite these differences, beliefs justified by visual blur seem to be about the functioning of our perception and not about properties of external objects. In consequence, the epistemic role of blurred experiences seems to be similar to those of typical interoceptive experiences.

Second, occurrences of visual blur motivate certain actions directed toward the visual system and not toward external objects. In particular, in cases of global blur, these actions are aimed toward restoring clarity of vision. They may be quite automatic, like blinking, squinting, or rubbing the eyes, but may also involve complex conceptualization, as involved in putting on glasses or seeking medical attention. Such a variety of responses occur also in case of other interoceptive experiences. For instance, some acute painful experiences automatically evoke bodily movement, like removing one's hand from a painful stimulus, while chronic pain often motivates a search for professional help.

It should be noted that the proposal above is consistent with the possibility of mistaking a blurred experience with an exteroceptive experience, like that of a fuzzy object. Even if the phenomenology of visual blur provides a justification for beliefs about vision, such a mistake can happen in at least two ways. First, it may happen due to a higher-order deficit. For instance, while one may have an experience with the phenomenal character characteristic of blurred vision, due to a lack of attentional resources one may not correctly recognize that it is an experience involving visual blur. Second, mistaking a blurred experience as an experience as of a fuzzy object may occur when, due to a lack of sufficient information, the situation is ambiguous for the perceptual mechanisms. As postulated by Schroer (2002), distinguishing between blur and fuzziness requires certain heuristics in which the perceptual system compares edges positioned in different fragments of the visual field. If such heuristics cannot be applied successfully, for instance, because the ability to change the eyes' focus and register subsequent modifications in acuity are somehow limited, the resulting perceptual phenomenal character may itself be ambiguous between that characteristic of blurred vision and of exteroceptive fuzziness.

The similarities between visual blur and interoceptive experiences suggest that blurred experiences have an interoceptive aspect which presents some state of the visual system. In terms of representationalist theories of perception it may be stated that such experiences possess some interoceptive content. Of course, blurred vision is not purely interoceptive. In fact, as demonstrated by the amplification argument (Bourget 2015), transition from clear to blurred vision involves a certain loss, but not complete disappearance, of usual visual exteroceptive content. In consequence, I propose that two types of content should be postulated to explain the phenomenon of blurred vision. The first is the exteroceptive visual content which characterizes perceived, external entities. When vision becomes blurred, some of this content is lost. The second is the interoceptive visual content which characterizes a state of the visual system. When vision becomes blurry, a phenomenal blurry quality appears which is determined by such interoceptive content. 
Furthermore, because there is a phenomenal continuity between clear vision and severely blurred vision, I propose a more general thesis that all visual experiences have both interoceptive and exteroceptive content. Structurally, this proposal is analogous to Pace's (2007) layered view of visual content. However, Pace proposed that visual states have exteroceptive content and content characterizing mental entities such as the visual field. Instead, according to my theory, both types of content characterize mind-independent entities: external objects and the visual system. The proposed theory is consistent with the representationalist thesis, as it allows for representationalist treatment of the distinction between blurred and clear vision and the distinction between blurred vision and experiences of fuzzy objects. First, the phenomenal transition between clear and blurred vision entails a change in the interoceptive content of a visual experience and a loss of some exteroceptive content. Second, the phenomenal difference between visual blurriness and visual fuzziness also corresponds to a difference in representational content. While visual blurriness presents a state of vision and is associated with a loss of exteroceptive content, visual fuzziness is a property exteroceptively attributed to edges of external things. The interoceptive theory is also consistent both with the metaphysical and the phenomenal version of the transparency thesis formulated in terms of mindindependentness. According to the metaphysical transparency thesis, we are in fact visually aware only of mind-independent objects and properties. This is consistent with the interoceptive theory, as it postulates that visual experiences have exteroceptive content characterizing external entities and interoceptive content characterizing the visual system. The phenomenal transparency thesis states that it always seems to us that we are visually aware of mind-independent objects and properties. The interoceptive theory postulates that this thesis is plausible due to the positive aspects of blurred experiences: visual blur seems to be about the visual system, as it leads to beliefs and actions concerning the visual apparatus.

\subsection{Content of depth and global blur}

Nevertheless, there are certain aspects of the theory described above which require further explication. First, according to my theory, both clear and blurred experiences have some interoceptive content. However, while there is a specific phenomenal quality in case of blurred experiences which corresponds to the interoceptive content, such a salient quality does not seem to be present in clear experiences. Thus, a question arises as to whether the interoceptive theory has resources to accommodate this phenomenal disunity between blurred and clear experiences. Second, the motivational aspect that was used in justifying the interoceptive character of visual blur is more apparent in cases of global blur than depth blur. In consequence, one may doubt whether the interoceptive theory of blurred vision is able to account for depth blur. Third, a plausible theory of blurred vision should not only postulate that there is some interoceptive content of visual states but should also provide some sensible idea concerning what this content is; i.e., what is presented about visual system in clear and blurred experiences. Fourth, while visual blur is not attributed to the external environment, it seems to have a certain spatial aspect (see Mehta 2013). In particular, in globally blurred experiences all objects 
look blurry, but in others that involve depth but not global blur, only distal (or only proximal) objects are seen in a blurry way. Hence, one may ask whether the interoceptive theory can plausibly explain these spatial differences.

To answer these questions, let us as a starting point consider a particular class of interoceptive experiences: proprioceptive experiences presenting the position of bodily parts. It is commonly claimed that usually proprioceptive experiences are only peripherally present in our consciousness unless something attracts attention toward a bodily part (e.g., Elder 2013; Hochstetter 2016; Lana 2017). For instance, in a normal situation the phenomenology of having a leg bend in a particular way seems to be less phenomenally salient than in cases of painful or tactile experiences. Similarly, when conducting common movements, we do not have a salient phenomenology of specific positions of bodily parts at subsequent moments (see Marcel 2003). Furthermore, such peripheral proprioceptive experiences are not usually associated with any strong motivational force. However, the phenomenal status of proprioceptive experiences changes when something goes wrong. For instance, when one fails to conduct an action, like grabbing an item, or when one's bodily position is uncomfortable (Kinsbourne 1995). In such cases, proprioceptive experiences become more salient and their motivational aspect increases. Sometimes, when proprioceptive processing is severely disturbed by neurological damage, a person may have a feeling of 'disembodiment' or experience a body part as 'alien,' which is severely disturbing and occupies a central place in consciousness (Ford and Smith 2006). The example of proprioceptive experiences shows that there are interoceptive experiences that have two important characteristics: (a) in normal cases they are not phenomenally salient and are not associated with strong motivational aspect, but (b) their saliency and motivational aspect are likely to be stronger when an experience presents some form of disorder.

Having the above features of certain interoceptive experiences in mind, let us investigate in a more detailed way the phenomenon of depth blur. In fact, the majority of visual experiences we intuitively categorize as 'clear' involve some form of depth blur, as depending on how our eyes are focused, not all objects are perceived with the same acuity. In other words, usual visual experiences contain some combination of phenomenal clearness and phenomenal blurriness. More precisely, the phenomenal combinations of clearness and depth blur occurring in ordinary experience are determined by two factors.

To demonstrate it, let's consider a visual scene composed of a proximally positioned apple and a distally positioned pear. The first factor is eye focus: when one's eyes are focused on the apple, the pear looks blurry, and conversely, when one's eyes are focused on the pear, the apple looks blurry. Without any changes in the composition of the visual scene, phenomenal combinations of depth blur and clearness may be modified by differences in eye focus that lead to distinct distributions of acuity. The second factor concerns modifications of the visual scene without changing the eye focus. For instance, if one's eyes are focused on the proximal apple, the distal pear looks blurry, but there is no phenomenally salient visual blur in empty space around the pear. However, if without changing the eye focus another object, perhaps an orange, is positioned next to the pear, it will be 
saliently blurred. In consequence, the overall combination of phenomenal clearness and depth blur would change without any modification of eye focus.

Relying on the above considerations, I propose that the interoceptive content characterizes a state of the visual system: a distribution of visual acuity in relation to eye focus. More precisely, as each eye focus is associated with a distribution of visual acuity, the interoceptive content of visual experiences can be described as a triple: < acuity distribution $D_{x}$, eye focus $F_{x}$, matching relation $R>$. The relational element $R$ specifies whether the distribution of acuity matches the eye focus. For instance, if one's eyes are focused on a proximal object and distal objects are blurred, then the distribution of acuity matches eye focus. However, if one's eyes are focused on a proximal object but all objects, both distal and proximal, are blurred, then matching does not occur. Later, I refer to this element in order to distinguish depth blur from global blur. The distribution of acuity specifies what acuity the visual system has for each perceived entity, like an object, an object's part, or an empty place, and can be modeled as a conjunction: visual system has acuity $A_{x}$ for entity $E_{y}$ and $\ldots$ and visual system has acuity $A_{n}$ for entity $E_{m}$, where $A_{x}$ and $A_{n}$ represent various levels of acuity and $E_{y}$ and $E_{m}$ represent perceived entities.

The phenomenal combinations of clearness and depth blur supervene on the proposed interoceptive content. For illustration, let us consider the earlier example in which one changes eye focus from a proximal apple to a distal pear. The resulting phenomenal change in combination of clearness and depth blur is determined by a change in interoceptive visual content. The eye focus and the distribution of acuity changes, and while earlier interoceptive content contained elements such as visual system has high acuity for apple-entity, visual system has low acuity for pear-entity, now it contains the following elements: visual system has low acuity for appleentity, visual system has high acuity for pear-entity. Similarly, when a distal orange is added next to a distal pear, while one's eyes are constantly focused on a proximal apple, the occurring phenomenal change in combination of clearness and depth blur is also associated with a change in the interoceptive content. A new element of content appears, visual system has low acuity for orange-entity, which replaces an earlier element specifying that the visual system has equally low acuity for some empty place (now occupied by an orange). Furthermore, changes in interoceptive content have consequences for exteroceptive content, as the transition from higher to lower acuity is associated with a loss of exteroceptive content.

Phenomenal combinations of clearness and depth blur are normal occurrences which depend in an important way on the manner in which eyes are focused. In consequence, similar to the case of normal proprioceptive experiences, they are not particularly salient and are not associated with a strong motivational force. However, there is a special type of blurred experiences in which acuity is lowered independently from the eye focus: occurrences of global blur. In usual experiences involving depth blur, the distribution of acuity changes together with eye focus; for instance, the interoceptive content may be at first $\left\langle D_{1}, F_{1}, R\right\rangle$ and then $\left\langle D_{2}, F_{2}\right.$, $R>$, where matching relation $R$ represents that in both cases acuity distribution matches eye focus. On the other hand, when global blur occurs, interoceptive content is such that despite changes in eye focus, the distribution of acuity remains 
the same or is lowered in a way that is unexpected, for example content $<D_{l}, F_{1}$, $R>$ changes to $\left\langle D_{1}, F_{2}, \sim R>\right.$, where matching relation $\sim R$ represents that there is no matching between eye focus and distribution of acuity. A loss of acuity occurring independently from changes in eye focus signals a disorder in the visual system's function, and so global blur is phenomenally salient and likely to motivate actions directed toward the eyes.

The above considerations allow me to address the four questions asked earlier. First, the phenomenal features associated with interoceptive content are more salient in cases of global blur than in case of combinations of clearness and depth blur because the phenomenology of global blur is characteristic of disorders of the visual system. Second, for the same reasons, globally blurred experiences typically have a stronger motivational component. Third, in both cases of combinations of clearness with depth blur and of global blur, the interoceptive content is of the same type. All of these phenomena interoceptively present acuity of vision in relation to eye focus, where global blur is associated with a special situation in which acuity is lowered independently of eye focus. Fourth, the specification of interoceptive content allows explanation of why blurred experiences may differ in spatial characteristics of visual blur: it is so because interoceptive content characterizes visual acuity in relation to eye focus, and different ways of focusing one's eyes determines whether distal or proximal objects are perceived as blurry.

Furthermore, by specifying the interoceptive content of blurred experiences, the phenomenon of blurred vision can be distinguished from that of peripheral vision. The blurriness-related phenomenology interoceptively presents the acuity of vision in relation to eye focus and is associated with some loss of exteroceptive content. Peripheral vision also plausibly has some interoceptive content and involves loss of exteroceptive content. However, it is unlikely that the peripheral interoceptive content concerns changes in acuity resulting from eye focus, as a change in focus has little effect on how peripheral information is processed. While proper investigation of this would require another paper, it is probable that the specific phenomenology of peripheral vision interoceptively presents that the stimuli is processed in a less detailed way due to eyes' position rather than focus.

Earlier, I have noted that the interoceptive theory is consistent both with the representationalist thesis and certain strong readings of the transparency thesis. Furthermore, it is also unthreatened by problems which are typical for theories of blurred vision described in Sect. 2. First, the interoceptive solution preserves the intuition that visual blur is not attributed to external entities, as blur-related changes regarding exteroceptive content consist only of loss of content and not the attribution of any additional property. Second, according to interoceptive theory there is no need to postulate that the content of blurred experiences is in some way internally inconsistent, or that there is no phenomenal difference between blurred experiences and clear experiences presenting fuzzy items. Finally, the proposed interoceptive theory is able to account for the epistemic role of visual blur and its motivational aspect. 


\section{Conclusions}

I have proposed a new theory of blurred vision according to which visual experiences have two types of content: exteroceptive content, characterizing external entities, and interoceptive content, characterizing the state of the visual system. More specifically, blurriness-related phenomenology interoceptively presents acuity of vision in relation to eye focus. When vision becomes globally blurred, some exteroceptive content is lost and the visual system is presented as having lowered acuity independent of how one's eyes are focused. This is experienced as an abnormal state and is often connected with motivational force. On the other hand, normal experiences involve some phenomenal combination of clearness and depth blur that is largely determined by eye focus. Such experiences are not connected with strong motivational force but still possess an interoceptive aspect presenting a distribution of visual acuity in relation to eye focus. The proposed theory is consistent with the representationalist thesis that phenomenal character supervenes on representational content and with the strong transparency thesis formulated in terms of mind-independentness. Furthermore, the interoceptive approach is free of the controversial assumptions adopted by other theories of blurred vision and is able to explain the epistemic and motivational role of visual blur.

Acknowledgements The author would like to thank the anonymous reviewer for comments concerning the paper. The early version of the paper was presented during SemDok seminar at Jagiellonian University.

Funding The work was supported by the National Science Center (Poland) Grant 2018/31/D/HS1/00363.

\section{Compliance with ethical standards}

Conflict of interest The author declares that he has no conflict of interest.

Ethical approval I comply with the Ethical Standards of "Philosophical Studies".

Informed consent and animal welfare The research did not involve human participants or animals.

Open Access This article is licensed under a Creative Commons Attribution 4.0 International License, which permits use, sharing, adaptation, distribution and reproduction in any medium or format, as long as you give appropriate credit to the original author(s) and the source, provide a link to the Creative Commons licence, and indicate if changes were made. The images or other third party material in this article are included in the article's Creative Commons licence, unless indicated otherwise in a credit line to the material. If material is not included in the article's Creative Commons licence and your intended use is not permitted by statutory regulation or exceeds the permitted use, you will need to obtain permission directly from the copyright holder. To view a copy of this licence, visit http:// creativecommons.org/licenses/by/4.0/.

\section{References}

Allen, K. (2013). Blur. Philosophical Studies, 162, 257-273. 
Avila, I. (2016). Is bodily awareness a form of perception? Phenomenology and Cognitive Science, 16(3), 337-354.

Aydede, M. (2017). Is the experience of pain transparent? Introspecting phenomenal qualities. Synthese, 196, 677-708.

Bach, K. (1997). Engineering the mind. Philosophy and Phenomenological Research, 57(2), 459-468.

Bain, D. (2003). Intentionalism and pain. The Philosophical Quarterly, 53(213), 502-523.

Boghossian, P. A., \& Velleman, J. D. (1989). Colour as a secondary quality. Mind, 90(389), 81-103.

Bourget, D. (2015). Representationalism, perceptual distortion and the limits of phenomenal concepts. Canadian Journal of Philosophy, 45(1), 16-36.

Crane, T. (2006). Is there a perceptual relation? In T. S. Gendler \& J. Hawthorne (Eds.), Perceptual experience (pp. 126-146). Oxford: Clarendon Press.

Crane, T., \& French, C. (2015). The problem of perception. The Stanford Encyclopedia of Philosophy (Spring 2017 Edition), E. N. Zalta (Ed.), https://plato.stanford.edu/archives/spr2017/entries/ perception-problem/.

Daly, C. (1994). Tropes. Proceedings of the Aristotelian Society, 94, 253-261.

Dretske, F. (2003). Experience as representation. Philosophical Issues, 13, 67-82.

Elder, A. (2013). Proprioception, anosognosia, and the richness of conscious experience. Journal of Consciousness Studies, 20(3-4), 83-101.

Ford, J., \& Smith, D. W. (2006). Consciousness, self, and attention. In U. Kriegel \& K. Williford (Eds.), Self-representational approaches to consciousness (pp. 353-377). Cambridge, MA: MIT Press.

French, C. (2015). Idiosyncratic perception. The Philosophical Quarterly, 66(263), 391-399.

Fridland, E. (2011). The case for proprioception. Phenomenology and Cognitive Sciences, 10, 521-540.

Gow, L. (2019). Everything is clear: All perceptual experiences are transparent. European Journal of Philosophy, 27(2), 412-425.

Heil, J. (2003). From an ontological point of view. Oxford: Oxford University Press.

Hochstetter, G. (2016). Attention in bodily awareness. Synthese, 193(12), 3819-3842.

Kinsbourne, M. (1995). Awareness of one's own body: An attentionnal theory of its nature, development, and brain basis. In J. L. Bermudez, T. Marcel, \& N. Eilan (Eds.), The body and the self (pp. 205-224). Cambridge, MA: MIT Press.

Klein, C. (2012). Imperatives, phantom pains, and hallucination by presupposition. Philosophical Psychology, 25(6), 917-928.

Lana, K. (2017). The missing pieces in the scientific study of bodily awareness. Philosophical Psychology, 30(5), 571-593.

Marcel, A. (2003). The sense of agency: Awareness and ownership of action. In J. Roessler \& N. Eilan (Eds.), Agency and self-awareness: Issues in philosophy and psychology (pp. 48-93). Oxford: Oxford University Press.

Martin, M. (2002). The transparency of experience. Mind \& Language, 17(4), 376-425.

Martin, M. (1993). Sense modalities and spatial properties. In N. Eilan, R. McCarthy, \& B. Brewer (Eds.), Spatial representation: Problems in philosophy and psychology (pp. 206-218). Oxford: Oxford University Press.

Mattens, F. (2016). The sense of touch: From tactility to tactual probing. Australasian Journal of Philosophy, 95(4), 688-701.

Mattens, F. (2013). Perception and representation: Mind the hand! In Z. Radman (Ed.), The hand, an organ of the mind: What the manual tells the mental (pp. 159-184). Cambridge, MA: The MIT Press.

Mehta, N. (2013). Beyond transparency: The spatial argument for experiential externalism. Philosophers' Imprint, 13(8), 1-19.

Nanay, B. (2018). Blur and perceptual content. Analysis, 78(2), 254-260.

O'Sullivan, B., \& Schroer, R. (2012). Painful reasons: Representationalism as a theory of pain. The Philosophical Quarterly, 62(249), 729-758.

Pace, M. (2007). Blurred vision and the transparency of experience. Pacific Philosophical Quarterly, 88, $328-354$.

Pautz, A. (2010). Why explain visual experience in terms of content? In B. Nanay (Ed.), Perceiving the world (pp. 254-309). Oxford: Oxford University Press.

Ratcliffe, M. (2008). Touch and situatedness. International Journal of Philosophical Studies, 16(3), 299-322.

Richardson, L. (2010). Seeing empty space. European Journal of Philosophy, 18(2), 227-243. 
Richardson, L. (2011). Bodily sensation and tactile perception. Philosophy and Phenomenological Research, 86(1), 134-154.

Schroer, R. (2002). Seeing it all clearly: The real story on blurry vision. American Philosophical Quarterly, 39(3), 297-301.

Schwenkler, J. (2013). The objects of bodily awareness. Philosophical Studies, 162, 465-472.

Siegel, S., \& Silins, N. (2015). The epistemology of perception. In M. Matthen (Ed.), The Oxford handbook of philosophy of perception. Oxford: Oxford University Press. https://doi.org/10.1093/ oxfordhb/9780199600472.013.040.

Slaby, J. (2008). Affective intentionality and the feeling body. Phenomenology and Cognitive Science, 7 , $429-444$.

Smith, A. D. (2008). Translucent experiences. Philosophical Studies, 140, 197-212.

Smith, A. D. (2011). Tastes, temperatures, and pains. In F. Macpherson (Ed.), The senses: Classical and contemporary philosophical perspectives (pp. 341-354). Oxford: Oxford University Press.

Tye, M. (2002). Representationalism and the transparency of experience. Nous, 36, 137-151.

Tye, M. (2003). Blurry images, double vision, and other oddities: New problems for representationalism? In Q. Smith \& A. Jokic (Eds.), Consciousness. New philosophical essays (pp. 7-32). Oxford: Clarendon Press.

de Vignemont, F. (2017). Pain and touch. The Monist, 100, 465-477.

de Vignemont, F., \& Massin, O. (2013). Touch. In M. Matthen (Ed.), The Oxford handbook of philosophy of perception. Oxford: Oxford University Press. https://doi.org/10.1093/oxfordhb/9780199600472. 013.017

Publisher's Note Springer Nature remains neutral with regard to jurisdictional claims in published maps and institutional affiliations. 